\title{
Practice Patterns for Eosinophilic Esophagitis Patients in Busan and Gyeongnam: A Korean Multicenter Database Study
}

\begin{abstract}
Su Jin Kim, ${ }_{1}^{1}$ Moo In Park, ${ }^{2 *}$ Gwang Ha Kim, ${ }^{3}$ Moon Won Lee, ${ }^{3}$ Kyoungwon Jung, ${ }^{2}$ Jin Lee, ${ }^{4}$ Sang Young Seol, ${ }^{5}$ Sam Ryong Jee, ${ }^{5}$ Hong Sub Lee, ${ }^{5}$ Jin Seok Jang, ${ }^{6}$ and Jae Hwang Cha ${ }^{6}$; Busan, Ulsan, Gyeongnam Society of Neurogastroenterology and Motility

${ }^{I}$ Department of Internal Medicine, Pusan National University School of Medicine, and Biomedical Research Institute, Pusan National University Yangsan Hospital, Yangsan, Gyeongsangnam-do, Korea; ${ }^{2}$ Department of Internal Medicine, Kosin University College of Medicine, Busan, Korea; ${ }^{3}$ Department of Internal Medicine, Pusan National University School of Medicine, and Biomedical Research Institute, Pusan National University Hospital, Busan, Korea; ${ }^{4}$ Department of Internal Medicine, Inje University College of Medicine, Haeundae Paik Hospital, Busan, Korea; ${ }^{5}$ Department of Internal Medicine, Inje University College of Medicine, Busan Paik Hospital, Busan, Korea; and ${ }^{6}$ Department of Internal Medicine, Dong-A University Hospital, Busan, Korea
\end{abstract}

\section{Background/Aims}

The prevalence of eosinophilic esophagitis is increasing in Korea and there are few single-center studies regarding eosinophilic esophagitis in Korea. In particular, data about management for eosinophilic esophagitis are lacking. We aim to evaluate the practice patterns, including initial treatment and response, in the Busan city and Gyeongnam province area.

\section{Methods}

We retrospectively reviewed medical records to gain data on patient characteristics, medication, endoscopic images, and esophageal biopsy results. From January 2009 to December 2019, a total of 42 patients were diagnosed with eosinophilic esophagitis.

\section{Results}

The mean age was 50.7 (from 22 to 81 ) years and the cohort was predominantly male $(78.6 \%, 33 / 42)$. The proton pump inhibitor was the preferred treatment as an initial trial for $64.3 \%$ (27/42) of patients, followed by swallowed topical steroids $(16.7 \%, 7 / 42)$. Clinical improvement after proton pump inhibitor therapy was achieved in $88.9 \%$ (24/27) of patients. Two patients who did not achieve improvement showed a clinical and endoscopic response after swallowed topical steroids treatment. No patient received diet elimination or balloon dilatation therapy.

\section{Conclusions}

The treatment response of eosinophilic esophagitis was good in Busan city and Gyeongnam province area in Korea. Proton pump inhibitor therapy was the preferred and most effective treatment for eosinophilic esophagitis as the initial therapy.

(J Neurogastroenterol Motil 2021;27:71-77)

Key Words

Eosinophilic esophagitis; Proton pump inhibitors; Steroids

Received: April 9, 2020 Revised: May 19, 2020 Accepted: June 8, 2020

(.) This is an Open Access article distributed under the terms of the Creative Commons Attribution Non-Commercial License (http://creativecommons. org/licenses/by-nc/4.0) which permits unrestricted non-commercial use, distribution, and reproduction in any medium, provided the original work is properly cited.

${ }^{*}$ Correspondence: Moo In Park, MD, PhD

Department of Internal Medicine, Kosin University College of Medicine, 262 Gamcheon-ro, Seo-gu, Busan 49267, Korea Tel: +82-51-990-6719, Fax: +82-51-990-5055, E-mail: mipark@kosinmed.or.kr 


\section{Introduction}

Eosinophilic esophagitis (EoE) is a chronic, immune/antigenmediated, esophageal disorder that typically presents with dysphagia or food impaction related with esophageal dysfunction and is characterized by eosinophilic predominant inflammation. ${ }^{1}$ Inflammation in EoE characteristically consists of $\geq 15$ eosinophils per high power field (HPF) on an esophageal biopsy. The gastrointestinal eosinophilia is limited to the esophagus and other causes of esophageal eosinophilia should be ruled out. ${ }^{2}$ The incidence of EoE appears to be rising with increased recognition of the disorder. ${ }^{3}$ Recent Korean studies have also reported that the EoE incidence appears to have increased considerably during the observation period. ${ }^{4,5}$

As inflammation in EoE might persist without treatment, patients develop esophageal fibrostenosis caused by progressive esophageal remodeling. ${ }^{6}$ The goal of treatment is symptomatic relief, ideally with histological remission. The initial management of EoE includes proton pump inhibitors (PPI), swallowed topical steroids (STS), and elimination diet. ${ }^{1}$ PPI may benefit patients with EoE either by reducing acid production, or by other anti-inflammatory effects. ${ }^{2}$ STS have shown effectiveness on the induction and maintenance of EoE. ${ }^{7,8}$ The elimination diet enables the examination of specific food allergies that commonly cause hypersensitivity in the general population. ${ }^{9}$ Future studies are needed to compare the effectiveness between these therapies. As the initial regimen to treat $\mathrm{EoE}$ remains undefined, the approach to patients is heterogeneous across providers. $^{2}$ In addition, the optimal second-line treatment modality after failure of the first-line therapy is also uncertain. To date, substantial variability in adherence to guidelines regarding practice patterns in EoE are documented. ${ }^{10-12}$

The management patterns of EoE have not been reported in Asian countries. Recent guidelines suggest that PPI should be considered as an initial treatment because of the low cost, good safety profile, and convenience; and the response of any therapy should be checked by a follow-up endoscopy with a biopsy. ${ }^{2}$ In the present study, we aim to evaluate the practice patterns including treatment, efficacy of therapy, and follow-up in the Busan city and Gyeongnam province area in Korea.

\section{Materials and Methods}

The medical records of patients (age more than 18 years) who received endoscopy in 6 tertiary care hospitals (Kosin University College of Medicine, Pusan National University Hospital, Busan
Paik Hospital, Dong-A University Hospital, Haeundae Paik Hospital, and Pusan National University Yangsan Hospital) in Busan and Gyeongnam, Korea, between January 2009 and December 2019 were retrospectively reviewed. Among 8 referral centers in Busan and Gyeongnam, $75.0 \%$ (6/8) of hospitals participated in this study. Busan-Gyeongnam is the second metropolitan area after the Seoul capital area, has a population of 7 million, as of 2010. It is located in the southeast of South Korea.

During the study period, EoE was confirmed by performing endoscopy with an esophageal biopsy. All esophageal biopsy reports of 6 center during 10 years were analyzed. Consequently, a total of 42 patients diagnosed with $\mathrm{EoE}$ at the 6 hospitals were included. The following data were collected and analyzed from medical records: patient characteristics, treatment details including medications, dietary and endoscopic interventions, endoscopic images and esophageal biopsy results, presence of allergies, and season of diagnosis. This study was approved by the Institutional Review Board of each hospital (05-2019-150).

\section{Diagnosis of Eosinophilic Esophagitis}

Esophageal biopsy specimens were obtained from the mid to proximal and distal ( $5 \mathrm{~cm}$ above the gastroesophageal junction) esophagus. ${ }^{13}$ EoE was defined when all of the following criteria were fulfilled: (1) a peak value of 15 or more eosinophils per HPF, (2) endoscopic abnormality or symptoms related to esophageal dysfunction, and (3) exclusion of other causes that could potentially contribute to esophageal eosinophilia. ${ }^{2}$ All of the biopsy specimen slides were reviewed by specialized pathologists of each center.

\section{Endoscopic Evaluation}

Endoscopic examinations with biopsy were performed by the endoscopy faculty or gastroenterology fellows under supervision of endoscopists who had the experience of over 10000 endoscopic examinations. Endoscopic features suggestive of EoE were classified using the endoscopic reference score system (EREFS). ${ }^{14}$ Edema was graded as 0 (absent) or 1 (present, loss of vascular markings). Rings were graded as 0 (absent), 1 (mild, circumferential ridges), 2 (moderate, distinct rings that did not impair passage of a standard endoscope), or 3 (severe, distinct ring that did not allow a standard endoscope to pass). Exudates were grade as 0 (absent), 1 (mild, covering $<10 \%$ of the esophageal mucosa), or 2 (severe, involving $>10 \%$ ). Furrows were graded as 0 (absent) or 1 (present). Strictures were graded as 0 (absent) or 1 (present) (Fig. 1). 


\section{Definition}

Clinical response was defined as the remission or improvement of symptoms related with esophageal dysfunction. Endoscopic response was defined as the improvement in EREFS or EREFS < 2. ${ }^{15}$ Histological response was defined as an eosinophil peak count of $<15$ eosinophils/HPF at all esophageal levels on the follow-up biopsy. ${ }^{16}$ Lack of response was defined as persistence or worsening of symptoms combined with no improvement of endoscopic and histologic disease activity.

\section{Statistical Methods}

Categorical variables were analyzed using the chi-square test or Fisher's exact test and continuous variables were analyzed using the Student's $t$ test. The Kruskal-Wallis test was used to determine whether there is a significant difference in the incidence of $\mathrm{EoE}$ with 3 years of interval. A $P$-value $<0.05$ was considered statistically significant. Statistical calculations were performed using the Statistical Package for the Social Sciences version 21.0 for Windows (IBM Corp, Armonk, NY, USA).

\section{Results}

During the study period, EoE was diagnosed in 42 patients at 6 tertiary care hospitals (Fig. 2). The mean age was 50.7 (from 22 to 81$)$ years and the cohort was predominantly male $(78.6 \%$, $33 / 42)$. The proportion of patients with allergies was $28.6 \%$ (12/42). There was no seasonal variation in the first diagnosis of EoE. Dysphagia was the most common symptom related with esophageal dysfunction $(38.1 \%, 16 / 42)$, followed by heartburn $(21.4 \%, 9 / 42)$,
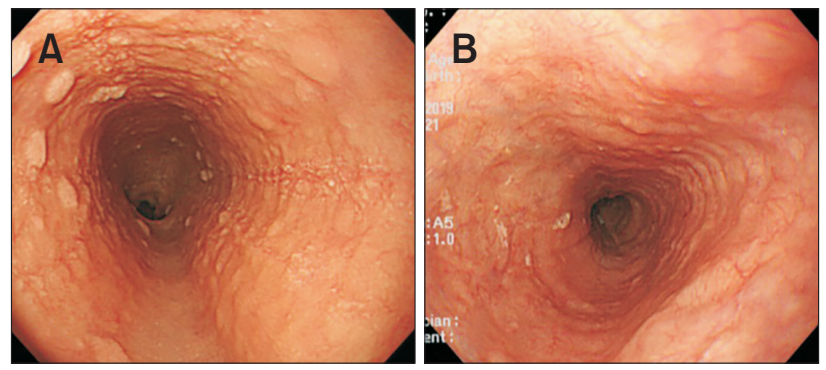

Figure 1. Endoscopic findings in eosinophilic esophagitis with application of the endoscopic reference score system. (A) E1 R0 E2 F1 S0: edema is grade as present (1); rings are graded as present (0); exudates are graded as severe (1); furrows are graded as mild (1); and stricture is graded as absent (0). (B) E1 R1 E1 F0 S0: edema is grade as present (1); rings are graded as mild (1); exudates are graded as mild (1); furrows are graded as absent (0); and stricture is graded as absent $(0)$. epigastric or abdominal pain $(14.3 \%, 6 / 42)$, food impaction $(4.8 \%$, $2 / 42$ ), and globus $(2.4 \%, 1 / 42)$ (Table 1$)$. In the endoscopic findings, furrows and edema were the most common $(57.1 \%, 24 / 42)$, followed by rings $(42.9 \%, 18 / 42)$, exudates $(14.3 \%, 6 / 42)$, and stricture $(9.5 \%, 4 / 42)$. The follow-up endoscopy interval (mean \pm standard deviation) was $10.6 \pm 5.7$ months.

\section{Choice of First- and Second-line Therapy for Eosinophilic Esophagitis}

PPIs were the preferred first-line therapy for $64.3 \%$ (27/42) of patients, followed by STS $(16.7 \%, 7 / 42)$. The duration (mean \pm standard deviation) of PPI and STS in first-line prescriptions were $172 \pm 226$ days and $98 \pm 87$ days, respectively. As a secondline therapy, 2 of 3 patients who did not achieve a clinical and endoscopic response after the PPI trial received STS treatment. Diet elimination or balloon dilatation therapy was not chosen as the firstor second-line treatment in this study. One-fifth of patients $(19.0 \%$, 8/42) did not received any treatment. Among the 8 non-treated patients, endoscopic improvement was found in 1 patient (12.5\%).

\section{Treatment Response for Eosinophilic Esophagitis}

A clinical response to PPI therapy was observed in $88.9 \%$ (24/27) of patients. An endoscopic response was achieved in $78.6 \%$ $(11 / 14)$ of patients who underwent the follow-up endoscopy after the PPI trial. Among 14 patients who received a follow-up endoscopy, esophageal biopsies were performed in 13. A histological response was observed in $84.6 \%$ (11/13) of patients who received follow-up biopsies. The interval of follow-up endoscopy was $10.6 \pm$ 5.7 months.

The clinical response rate of the first-line STS therapy was $100.0 \%(7 / 7)$. Approximately half of patients (4/7) received a

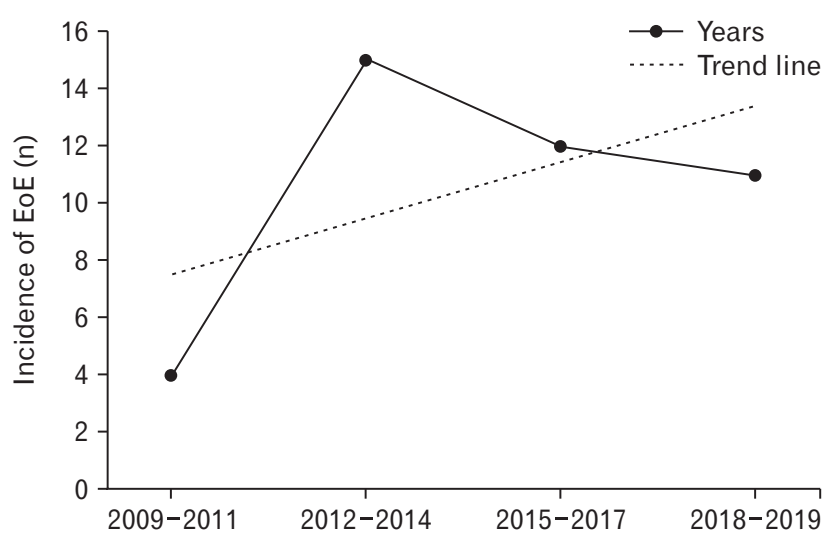

Figure 2. The changing incidence of eosinophilic esophagitis by 3 years of interval $(P=0.392)$. 
follow-up endoscopy with biopsy. An endoscopic and histological response was found in all patents (4/4). STS therapy after failure of PPI treatment showed a clinical and endoscopic response in all patients (2/2). Among 2 patients who received second-line STS therapy, a follow-up biopsy was performed in only 1 patient. A histological response was achieved in this patient.

\section{Comparison of Clinical Factors Between Therapy Choices for Eosinophilic Esophagitis}

The presence of esophageal symptoms was significantly different between non-treated and treated patients (Table 2). All non-

Table 1. Baseline Characteristics of the Study Population

\begin{tabular}{|c|c|}
\hline Characteristics & Total $(\mathrm{N}=42)$ \\
\hline Age (yr) & $51.6 \pm 15.1$ \\
\hline Male/female & $33 / 9(78.6 / 21.4)$ \\
\hline Alcohol & $16(38.1)$ \\
\hline Smoking & $8(19.0)$ \\
\hline \multicolumn{2}{|l|}{ Allergic disease } \\
\hline Allergic rhinitis & $4(9.5)$ \\
\hline Allergic dermatitis & $3(7.1)$ \\
\hline Asthma & $3(7.1)$ \\
\hline Food allergy & $2(4.8)$ \\
\hline \multicolumn{2}{|l|}{ Season to diagnose EoE } \\
\hline Spring/summer/autumn/winter & $\begin{array}{c}11(26.2) / 23(54.8) / \\
12(28.6) / 6(14.3)\end{array}$ \\
\hline \multicolumn{2}{|l|}{ Esophageal symptom } \\
\hline Dysphagia & $16(38.1)$ \\
\hline Heartburn & $9(21.4)$ \\
\hline Pain & $6(14.3)$ \\
\hline Globus & $1(2.4)$ \\
\hline Food impaction & $2(4.8)$ \\
\hline None & $15(35.7)$ \\
\hline \multicolumn{2}{|l|}{ EREFS } \\
\hline \multicolumn{2}{|l|}{ Edema } \\
\hline Grade 0 (absent)/1 (present) & $24(57.1) / 18(42.9)$ \\
\hline Rings & $\begin{array}{c}24(57.1) / 15(35.7) / \\
3(7.1) / 0(0.0)\end{array}$ \\
\hline $\begin{array}{l}\text { Grade } 0 \text { (none) } / 1 \text { (mild)/ } \\
2 \text { (moderate)/3 (severe) }\end{array}$ & $(57.1 / 35.7 / 15 / 7.1 / 0)$ \\
\hline \multicolumn{2}{|l|}{ Exudates } \\
\hline Grade 0 (none)/1 (mild)/2 (severe) & $36(85.7) / 1(2.4) / 5(11.9)$ \\
\hline \multicolumn{2}{|l|}{ Furrows } \\
\hline Grade 0 (absent)/1 (present) & $18 / 24(42.9 / 57.1)$ \\
\hline \multicolumn{2}{|l|}{ Stricture } \\
\hline Grade 0 (absent)/1 (present) & $38(90.5) / 4(9.5)$ \\
\hline Reflux esophagitis & $4(9.5)$ \\
\hline
\end{tabular}

EoE, eosinophilic esophagitis; EREFS, endoscopic reference score system. Data are presented as mean $\pm \mathrm{SD}$ or $\mathrm{n}(\%)$.
Table 2. Comparison of Clinical Factors Between Non-treated and Treated Patients

\begin{tabular}{lccc}
\hline \multicolumn{1}{c}{ Clinical factors } & $\begin{array}{c}\text { No treatment } \\
(\mathrm{n}=8)\end{array}$ & $\begin{array}{c}\text { PPI or STS } \\
(\mathrm{n}=34)\end{array}$ & P-value \\
\hline Age $(\mathrm{yr})$ & & & $>0.999$ \\
$<40$ & $2(25.0)$ & $8(23.5)$ & \\
$\geq 40$ & $6(75.0)$ & $26(76.5)$ & \\
Sex & & & 0.168 \\
Male & $8(100.0)$ & $25(73.5)$ & \\
Female & $0(0.0)$ & $9(26.5)$ & \\
Allergic disease & & & \\
None & $7(87.5)$ & $23(67.6)$ & \\
$\quad$ Present & $1(12.5)$ & $11(32.4)$ & \\
Esophageal symptoms & & & \\
$\quad$ None & $8(100.0)$ & $7(20.6)$ & \\
$\quad$ Present & $0(0.0)$ & $27(79.4)$ & \\
Endoscopic features & & & \\
Edema & $2(25.0)$ & $16(47.1)$ & 0.431 \\
Rings & $5(62.5)$ & $13(38.2)$ & 0.256 \\
Exudates & $1(12.5)$ & $5(14.7)$ & $>0.999$ \\
Furrows & $5(62.5)$ & $19(55.9)$ & $>0.999$ \\
Stricture & $0(0.0)$ & $4(11.8)$ & 0.572 \\
\hline
\end{tabular}

PPI, proton pump inhibitor; STS, swallowed topical steroid.

Data are presented as $n(\%)$.

Table 3. Comparison of Clinical Factors Between Proton Pump Inhibitor and Swallowed Topical Steroid Therapies as First Therapy

\begin{tabular}{|c|c|c|c|}
\hline Clinical factors & $\operatorname{PPI}(\mathrm{n}=27)$ & $\operatorname{STS}(n=7)$ & $P$-value \\
\hline Age (yr) & & & $>0.999$ \\
\hline$<40$ & $7(25.9)$ & $1(14.3)$ & \\
\hline$\geq 40$ & $20(74.1)$ & $6(85.7)$ & \\
\hline Sex & & & 0.644 \\
\hline Male & $19(70.4)$ & $6(85.7)$ & \\
\hline Female & $8(29.6)$ & $1(14.3)$ & \\
\hline Allergic disease & & & $>0.999$ \\
\hline None & $18(66.7)$ & $5(71.4)$ & \\
\hline Present & $9(33.3)$ & $2(28.6)$ & \\
\hline \multicolumn{4}{|l|}{ Esophageal symptoms } \\
\hline Dysphagia & $12(44.4)$ & $4(57.1)$ & 0.681 \\
\hline Heartburn & $7(25.9)$ & $2(28.6)$ & $>0.999$ \\
\hline Pain & $4(14.8)$ & $2(28.6)$ & 0.580 \\
\hline Globus & $1(3.7)$ & $0(0.0)$ & $>0.999$ \\
\hline Food impaction & $2(7.4)$ & $0(0.0)$ & $>0.999$ \\
\hline \multicolumn{4}{|l|}{ Endoscopic features } \\
\hline Edema & $15(55.6)$ & $1(14.3)$ & 0.090 \\
\hline Rings & $9(33.3)$ & $4(57.1)$ & 0.387 \\
\hline Exudates & $3(11.1)$ & $2(28.6)$ & 0.268 \\
\hline Furrows & $16(59.3)$ & $3(42.9)$ & 0.672 \\
\hline Stricture & $1(3.7)$ & $3(42.9)$ & 0.021 \\
\hline
\end{tabular}

PPI, proton pump inhibitor; STS, swallowed topical steroid. Data are presented as $\mathrm{n}(\%)$. 
treated patients (8/8) and one-fifth of treated patients (7/34) had no esophageal symptoms $(P<0.001)$. In patients who received therapy, the proportion of esophageal stricture was higher in STS $(42.9 \%, 3 / 7)$ than PPI $(3.7 \%, 1 / 27)(P=0.021)$ (Table 3$)$.

\section{Discussion}

This multicenter study of $42 \mathrm{EoE}$ cases from Busan city and Gyeongnam province in Korea assessed the management pattern and clinical factors affected by the choice of first-line therapy. PPI was the preferred option as an initial therapy $(64.3 \%, 27 / 42)$. Furthermore, STS was often chosen as an initial treatment especially in patients with esophageal stricture $(16.7 \%, 7 / 42)$. STS was regarded as the preferred option after failure of a PPI trial. Both PPI and STS showed effectiveness in EoE treatment. Half of patients without symptoms related to esophageal dysfunction (8/15) did not receive any treatment.

The role of PPI in the updated international consensus for EoE has evolved from exclusion of PPI-responsive EoE to treatment through the gastric acid inhibiting effect and anti-inflammatory effect unrelated to gastric acid. ${ }^{2}$ PPI is recommend as a potential early or initial therapy because of its low cost, good safety profile, and convenience. ${ }^{2}$ A recent Korean study demonstrated that PPI $(77.8 \%, 56 / 72)$ and STS $(5.6 \%, 4 / 72)$ were used as initial therapies in EoE. Another Korean study reported that PPI $(66.6 \%, 6 / 9)$ and STS $(22.2 \%, 2 / 9)$ were prescribed as first-line treatments. ${ }^{17}$ These results are consistent with the present study.

However, several previous studies have also reported that STS was the most common initial therapy. ${ }^{10,11}$ Our results demonstrated that the choice of STS as the first-line therapy was preferred in patients with esophageal stricture. This may be the reason for the difference in preferred initial therapy between Korea and other countries.

The prevalence of esophageal stricture is lower in Korean studies compared to other studies. ${ }^{4,10,11,17}$ First, the Korean National Gastric Cancer Screening Program using biannual endoscopy or barium study was performed in adults aged more than 40 years. It may lead to the early detection of fibrostenotic type EoE before progression to small caliber esophagus. Second, the biopsy based EoE diagnosis in this study can also contribute to the low incidence of esophageal strictures. Eosinophil counts are generally low in cases of advanced $\mathrm{EoE}$ with fibrosis. Third, the disease duration from diagnosis may be short compared to the Western countries as an awareness of EoE has been growing recently in Korea. This situation can also result in the low incidence of esophageal strictures.
In the present study, There were no patients who received dilation therapy. Two previous Korean studies also reported only 1 case of balloon dilation for esophageal stricture. ${ }^{4,17}$

Previous studies reported that the clinical and histological response rates ranged from $25.0 \%$ to $96.0 \%$ and $23.0 \%$ to $83.0 \%$, respectively. ${ }^{2}$ In this study, the clinical response rate of $88.9 \%$ (24/27) and histological response rate of $84.6 \%$ (11/13) were similar to the maximum range of reported rates. The concept of PPIresponsive EoE may increase the possibility to exclude the patients who showed response to PPI therapy in the previous studies. ${ }^{2}$ The exclusion of PPI-responsive EoE according to advances in the definition EoE might lead to a higher clinical and histological response to the PPI trial. All patients who received STS as first- or secondline therapy showed a $100.0 \%$ clinical (9/9) and histological (5/5) response. The requirement of esophageal dilation was reported as a predictor of poor response to STS in EoE patients. ${ }^{18,19}$ No patient needed dilation therapy in the present study, which may explain the high response rate of STS.

Other therapeutic approaches for $\mathrm{EoE}$ have been reported to be as effective and safe. Novel EoE specific steroid formulations have been developed to optimize mucosal deposition. Budesonide oral suspension in United States and the budesonide effervescent tablet in Europe have been approved and show effectiveness with negligible side effects. ${ }^{20,21}$ Biologics such as a monoclonal antibody against IL-13, dupilumab (monoclonal antibody to the alpha subunit of the IL-4 receptor), mepolizumab (monoclonal antibody against IL-5), reslizumab (IL-5 neutralizing antibody), and omalizumab (anti-IgE monoclonal antibody) are currently in phase II trials with EoE patients. ${ }^{22}$ Further studies for these novel therapies could result in personalized therapeutic strategies for EoE.

Finally, we found a gap in real practice and adherence to guidelines in monitoring the response to treatment. Guidelines recommend that follow-up endoscopy with biopsy should be performed to confirm the effectiveness of treatment. ${ }^{1,2}$ Half of the patients did not receive the follow-up endoscopy after first-line PPI (48.1\%, $313 / 27)$ and STS $(42.9 \%, 3 / 7)$ therapy. The decision for a followup endoscopy is based on the esophageal symptoms of $\mathrm{EoE}$ and this may contribute to the reason why the practice pattern differs from the guidelines. ${ }^{23}$ Esophageal biopsies during a follow-up endoscopy after treatment were not performed in 2 patients (one after initial PPI therapy, the other after second-line STS therapy). Changing the guidelines about $\mathrm{EoE}$ can result in non-adherence to the guidelines in monitoring the response to treatment. ${ }^{1,2,10}$

The strength of our study is that this is the first multi-center data analysis about real-world practice pattern for EoE in Asia. 
Previous Korean studies were single-center design, and Asian multi-center studies focused on the diagnosis and prevalence of EoE. ${ }^{4,5,17,24-27}$ Recent studies to evaluate the management for $\mathrm{EoE}$ were published in Western countries. ${ }^{10,23,28,29}$ The practice patterns of Busan-Gyeongnam, Korea, were different to those reported in Western countries. ${ }^{12,30,31}$ In the United States, gastroenterologists responded that topical steroids is the preferred therapy and endoscopy with biopsy is recommend by $72.0 \%$ of academic providers and $27.0 \%$ of private practices. ${ }^{31}$ German gastroenterologists responded that both PPI and STS were the preferred options. ${ }^{12}$ An international study including 14 European countries reported that there were geographical differences in the choice of first-line therapy. ${ }^{30} \mathrm{In}$ Korea, PPI was the most commonly used first-line approach, and follow-up endoscopy with biopsy was not performed routinely.

Our study has several potential limitations. First, this was a retrospective study. Patients received different PPIs, which could have affected the response to the treatment. The meta-analysis reported that lansoprazole (70.2\%) and rabeprazole (72.3\%) showed higher efficacy compared with omeprazole (53.5\%) and esomeprazole $(46.8 \%)$, regardless of the twice daily and once-daily doses. ${ }^{32}$ Second, the patients included in this multicenter study may not be representative of all Korean patients. There is a possibility of referral bias, because patients with severe disease may tend to visit specialized clinics in Seoul. This could have resulted in better clinical outcomes in the present study. Therefore, nationwide multicenter studies are needed to confirm this conclusion.

In conclusion, this study demonstrated that the response to PPI and STS therapies for EoE was favorable in Busan city and Gyeongnam province in Korea. PPI was the most common treatment used as the first-line therapy in this region. These results are similar to those of previous Korean studies. We found a trend in the use of STS in patients with esophageal stricture. Our findings regarding the adherence to guidelines suggest that physicians need to try to reduce the gap between guidelines and real practice in monitoring the treatment response.

\section{Financial support: None.}

\section{Conflicts of interest: None.}

Author contributions: Moo In Park designed the model and the computational framework; Su Jin Kim analysed the data and wrote draft manuscript; Moon Won Lee, Su Jin Kim, Kyoungwon Jung, Jin Lee, Hong Sub Lee, and Jae Hwang Cha collected the data; and Sang Young Seol, Sam Ryong Jee, Moo In Park, Gwang $\mathrm{Ha} \mathrm{Kim}$, and Jin Seok Jang contributed to the final version of the manuscript and supervised the project.

\section{References}

1. Lucendo AJ, Molina-Infante J, Arias Á, et al. Guidelines on eosinophilic esophagitis: evidence-based statements and recommendations for diagnosis and management in children and adults. United European Gastroenterol J 2017;5:335-358.

2. Dellon ES, Liacouras CA, Molina-Infante J, et al. Updated international consensus diagnostic criteria for eosinophilic esophagitis: proceedings of the AGREE conference. Gastroenterology 2018;155:1022-1033, e10.

3. Navarro P, Arias Á, Arias-González L, Laserna-Mendieta EJ, RuizPonce M, Lucendo AJ. Systematic review with meta-analysis: the growing incidence and prevalence of eosinophilic oesophagitis in children and adults in population-based studies. Aliment Pharmacol Ther 2019;49:1116-1125

4. Kim GH, Park YS, Jung KW, et al. An increasing trend of eosinophilic esophagitis in korea and the clinical implication of the biomarkers to determine disease activity and treatment response in eosinophilic esophagitis. J Neurogastroenterol Motil 2019;25:525-533.

5. Kim GH, Jung KW, Jung HY, et al. Diagnostic trends and clinical characteristics of eosinophilic esophagitis: a Korean, single-center database study. J Neurogastroenterol Motil 2018;24:248-254.

6. Dellon ES, Hirano I. Epidemiology and natural history of eosinophilic esophagitis. Gastroenterology 2018;154:319-332, e3.

7. Greuter T, Safroneeva E, Bussmann C, et al. Maintenance treatment of eosinophilic esophagitis with swallowed topical steroids alters disease course over a 5-year follow-up period in adult patients. Clin Gastroenterol Hepatol 2019;17:419-428, e6.

8. Murali AR, Gupta A, Attar BM, Ravi V, Kodura P. Topical steroids in eosinophilic esophagitis: systematic review and meta-analysis of placebo-controlled randomized clinical trials. J Gastroenterol Hepatol 2016;31:1111-1119

9. Zhan T, Ali A, Choi JG, et al. Model to determine the optimal dietary elimination strategy for treatment of eosinophilic esophagitis. Clin Gastroenterol Hepatol 2018;16:1730-1737, e2.

10. Huang KZ, Jensen ET, Chen HX, et al. Practice pattern variation in pediatric eosinophilic esophagitis in the carolinas EoE collaborative: a research model in community and academic practices. South Med J 2018;111:328-332.

11. Lucendo AJ, Arias Á, Molina-Infante J, et al. Diagnostic and therapeutic management of eosinophilic oesophagitis in children and adults: results from a Spanish registry of clinical practice. Dig Liver Dis 2013;45:562568.

12. Miehlke S, von Arnim U, Schlag C, et al. Clinical management of eosinophilic esophagitis - a nationwide survey among gastroenterologists in Germany. Z Gastroenterol 2019;57:745-752.

13. Gonsalves N, Policarpio-Nicolas M, Zhang Q, Rao MS, Hirano I. Histopathologic variability and endoscopic correlates in adults with eosinophilic esophagitis. Gastrointest Endosc 2006;64:313-319.

14. Hirano I, Moy N, Heckman MG, Thomas CS, Gonsalves N, Achem 
SR. Endoscopic assessment of the oesophageal features of eosinophilic oesophagitis: validation of a novel classification and grading system. Gut 2013;62:489-495.

15. Dellon ES, Cotton CC, Gebhart JH, et al. Accuracy of the eosinophilic esophagitis endoscopic reference score in diagnosis and determining response to treatment. Clin Gastroenterol Hepatol 2016;14:31-39.

16. Reed CC, Wolf WA, Cotton CC, et al. Optimal histologic cutpoints for treatment response in patients with eosinophilic esophagitis: analysis of data from a prospective cohort study. Clin Gastroenterol Hepatol 2018;16:226-233, e2.

17. Lee JH, Kim MJ, Kim JH, et al. Clinical analysis of primary eosinophilic esophagitis. J Neurogastroenterol Motil 2013;19:204-209.

18. Wolf WA, Cotton CC, Green DJ, et al. Predictors of response to steroid therapy for eosinophilic esophagitis and treatment of steroid-refractory patients. Clin Gastroenterol Hepatol 2015;13:452-458.

19. Eluri S, Selitsky SR, Perjar I, et al. Clinical and molecular factors associated with histologic response to topical steroid treatment in patients with eosinophilic esophagitis. Clin Gastroenterol Hepatol 2019;17:1081-1088, e2.

20. Lucendo AJ, Miehlke S, Schlag C, et al. Efficacy of budesonide orodispersible tablets as induction therapy for eosinophilic esophagitis in a randomized placebo-controlled trial. Gastroenterology 2019;157:74-86. e15.

21. Dellon ES, Katzka DA, Collins MH, et al. Safety and efficacy of budesonide oral suspension maintenance therapy in patients with eosinophilic esophagitis. Clin Gastroenterol Hepatol 2019;17:666-673, e8.

22. Greuter T, Hirano I, Dellon ES. Emerging therapies for eosinophilic esophagitis. J Allergy Clin Immunol 2020;145:38-45.

23. King J, Khan S. Eosinophilic esophagitis: perspectives of adult and pediatric gastroenterologists. Dig Dis Sci 2010;55:973-982.
24. Joo MK, Park JJ, Kim SH, et al. Prevalence and endoscopic features of eosinophilic esophagitis in patients with esophageal or upper gastrointestinal symptoms. J Dig Dis 2012;13:296-303.

25. Fujishiro H, Amano Y, Kushiyama Y, Ishihara S, Kinoshita Y. Eosinophilic esophagitis investigated by upper gastrointestinal endoscopy in Japanese patients. J Gastroenterol 2011;46:1142-1144.

26. Fujiwara Y, Sugawa T, Tanaka F, et al. A multicenter study on the prevalence of eosinophilic esophagitis and PPI-responsive esophageal eosinophilic infiltration. Intern Med 2012;51:3235-3239.

27. Shimura S, Ishimura N, Tanimura T, et al. Reliability of symptoms and endoscopic findings for diagnosis of esophageal eosinophilia in a Japanese population. Digestion 2014;90:49-57.

28. Laserna-Mendieta EJ, Casabona S, Savarino E, et al. Efficacy of therapy for eosinophilic esophagitis in real-world practice. Clin Gastroenterol Hepatol Published Online First: 25 Jan 2020. doi: 10.1016/ j.cgh.2020.01.024.

29. Peery AF, Shaheen NJ, Dellon ES. Practice patterns for the evaluation and treatment of eosinophilic oesophagitis. Aliment Pharmacol Ther 2010;32:1373-1382.

30. Tourlamain G, Garcia-Puig R, Gutiérrez-Junquera C, et al. Differences in management of eosinophilic esophagitis in Europe: an assessment of current practice. J Pediatr Gastroenterol Nutr 2020;71:83-90.

31. Chang JW, Saini SD, Mellinger JL, et al. Management of eosinophilic esophagitis is often discordant with guidelines and not patient-centered: results of a survey of gastroenterologists. Dis Esophagus 2019;32:doy133.

32. Lucendo AJ, Arias Á, Molina-Infante J. Efficacy of proton pump inhibitor drugs for inducing clinical and histologic remission in patients with symptomatic esophageal eosinophilia: a systematic review and metaanalysis. Clin Gastroenterol Hepatol 2016;14:13-22, e1. 